\title{
A Review on Epidimiology and Control Aspect of Dourine in Ethiopia
}

\section{Temesgen Kassa Getahun}

Ethiopian Institute of Agricultural Research Holeta Agricultural Research Center Animal Health Research Program, Holeta, Ethiopia

\section{Email address:}

temesgen.kassa@yahoo.com

\section{To cite this article:}

Temesgen Kassa Getahun. A Review on Epidimiology and Control Aspect of Dourine in Ethiopia. Animal and Veterinary Sciences. Vol. 7, No. 3, 2019, pp. 69-77. doi: 10.11648/j.avs.20190703.11

Received: March 28, 2019; Accepted: April 29, 2019; Published: June 13, 2019

\begin{abstract}
Dourine is a chronic or acute contagious disease of breeding equids which transmitted directly from animal to animal during coitus. It is caused by T. equiperdum which is rarely detected in the blood stream and normally localizes in the capillaries of the mucous membranes of the urogenital tract. The diagnosis of T. equiperdum is not straight forward and this can lead to difficulties in achieving reliable data on the prevalence and distribution of infections and in the implementations and monitoring of control programs of the disease. The only effective control of dourine remains strict control of breeding; good hygiene is essential during assisted mating because infection may be transmitted through contaminated fomites. So the main objective is to review the epidemiology and control method practiced in Ethiopia.
\end{abstract}

Keywords: Control, Dourine, Epidemiology, Equine (Horse) T. equiperdum

\section{Introduction}

In a country where is less developed modern transport and communication service, the natural choice rests on the use of human and pack animals. In developing country like Ethiopia, the contribution of equines in the energy scenario is of considerable significance. Ethiopia possesses about 2.70 million horses, 5.2 million donkeys, and 0.63 million mules; totally about 8.53 million equines. In this country the greatest numbers of equines are found in the central high land which corresponds to an average holding of one equine per rural family of five. The provision of transport through pack animals, drawing carts, as riding animals or taxi operation, almost certainly contributes more to the national economy [1].

Equine animals are extremely important in Ethiopian agriculture and for the national economy. Nearly $90 \%$ of agricultural operations depend on manual labor, and because of the rugged mountainous terrain of the country these animals are still the main method used to transport both people and agricultural products [1].

Those large equine populations in the country are affected by many diseases as in many developing countries. From those the one common disease is dourine, caused by trypanosoms. Trypanosome is a genus of kinetoplastids (class
Kinetoplastida), a monophyletic group of unicellular parasitic flagellate protozoa. The name is derived from the Greek trypano- (borer) and soma (body) because of their corkscrew-like motion. All trypanosomes are heteroxenous (requiring more than one obligatory host to complete life cycle) and most are transmitted via a vector. The majority of species are transmitted by blood-feeding invertebrates, but there are different mechanisms among the varying species. In an invertebrate host they are generally found in the intestine, but normally occupy the bloodstream or an intracellular environment in the mammalian host. Trypanosomes infect a variety of hosts and cause various diseases, including dourine which is caused by T.equiperdum in equine. According to [2] report major disease that affects horses in Africa, most of them are found in Ethiopia, are African horse sickness, epizootic lymphangitis, dourine, equine piroplasmosis, horse mange, rabies, glanders and ulcerative lymphangitis. The problem of dourine in Ethiopia has been recognized by local farmers for many years. However, the first official report of the disease in Ethiopia was made in 1980 when the Arsi Rural Development Unit asked the Tsetse and Trypanosomiasis Survey and Control Department to investigate a persistent disease problem in horses in the administrative regions of Arsi and Bale [3]. Since then, dourine has been found to be prevalent throughout the 
highlands of Ethiopia, particularly in the Arsi and Bale zones. Survey of horses in Arsi and Bale region of Ethiopia provides strong circumstantial evidence that T. equiperdum infectious are a potential threat to the well-being and productivity of the equine population [4]. T.equiperdium has the distribution of being the only trypanosome whose normal method of transmission is by coitus. The infection restricted in nature to equines, which it causes the disease dourine [5].

The diagnosis of T.equiperdium is not straight forward, owing to the low numbers of parasites present in the blood or tissue fluids and the frequent absence of clinical signs of disease and this can lead to difficulties in achieving reliable data on the prevalence and distribution of infections and in the implementation and monitoring of the disease in the endemic area of Ethiopia [6].

In view of this, the objective of this paper is to provide review on the importance of dourine, to give a brief account on the epidemiology and control of dourine and to give an overview of the available diagnostic techniques with respect to their uses and limitation.

\section{Dourine}

The disease has many synonyms depending on language of the individual describing the conditions, for example "ed dourine" in Arabic; "Mal de coit" in French; "Beschalkrankheit" or "Beschal seuche" in German and "Dourine" or covering disease in English [10]. In Ethiopia, the disease is known as commonly as "Lappessa Duda kuta" or Lappeessa hida kuta" which means back bone breaker. Since the clinical signs bear some resemblance to the human venereal disease it has sometimes been referred to as "equine syphilis" but the universal name is dourine [10].

\subsection{Etiology}

Throughout the world, the one common factor leading to the ill health, suffering and early demise of equines is the protozoan parasite causing dourine. Dourine is caused by Trypanosoma equiperdum, related morphologically and serologically to T. brucei, T. rhodesiense, and T. gambiense (of the subgenus Trypanozoon of the Salivarian section of organisms of the pathogenic genus Trypanosoma). Different strains of the parasite vary in pathogenicity [7]. It is a chronic or acute contagious disease of breeding equids that is transmitted directly from animal to animal during coitus. $T$. equiperdum is the only trypanosomosis that is not transmitted by an invertebrate vector. T. equiperdum differs from other trypanosomes in that it is primarily a tissue parasite that rarely invades the blood [4, 8]. Among the non-tsetse transmitted tyrpanosomiasis, dourine is included in list B of the OIE notifiable disease [9].

\subsubsection{Taxonomy}

The causative organism, trypanosomes, which are strictly parasitic are flagellar; belong to be the phylum of sorcomastogophora, the order of kinetoplastda, the family of trypanosomatidea and the genus of trypanosome, under the salivariyan group. The subgenus trypanozoon includes the pathogenic species T. evansi, T. brucei and T. equiperdum as indicated below in the table 1. T. brucei is further divided in to three subspecies; the animal pathogen T.b.brucei and trypanosome responsible for human sleeping sickness $T . b$. gambiense and T. b. rhodesiense [11].

Table 1. Taxonomy of T.equperdum.

\begin{tabular}{ll}
\hline Phylum & Protozoa \\
Class & Zoomastigophopra \\
Family & Trypanospomatidae \\
Genus & Trypanosomes \\
Subgenus & Trypanozoon(Brucei group) \\
Species & T.brucei, T.evnsi, T.equiperdum \\
Subspecies & T.b.brucei, T.b.gambiense, T.b.rhodesiense \\
\hline
\end{tabular}

Source: (Haore, 1992).

\subsubsection{Morphology}

The trypanosome causing dourine will seldom be found in the blood of equine, suffering from the disease, in ordinaly blood films. However, may be seen in centrifuged a aliquots of blood or in aspirated lymph from edematous fluids, vaginal washing or exudates. In stained preparation it is identical specimens of $T$. evansi (kinetoplastic forms), $T$. brucei and T.Rhodesiense [12]. The morphology of $T$. equiperdum is identical to the blood stream slender form of $T$. b.brucei, having a single free flagellum with long trypamastigote forms. Like T. evansi, T. equiperdum is usually monomorphic. However, it sometimes exhibits pleomorphism like T. evansi. Morphologically it is indistinguishable from T.evansi, being represented by this (Slender and intermediate) trypamastigotes possessing a freeflagellum although pleomorphic, stumpy protonuclear forms are recognized. Its length ranges from 15.5 to $36 \mathrm{~mm}$ with a mean length of $25.6-27.7 \mathrm{~mm}$, which is similar to that of $T$. evansi. As in T. evansi, the nucleus lies in the center of the body. The kinetoplast is very distinct and terminal or sub terminal in position with well-developed undulating membrane and free flagellum. The site of T. equperdum is likewise within the range of that of $T$. evansi with the length of different strains [12]. At the fine structural level, there are relatively more coated vesicles in the flagellar pocket of $\mathrm{T}$. equiperdum, compared with T. evansi. It becomes somewhat difficult to differentiate these two species with respect to the ultrastructural properties [7].

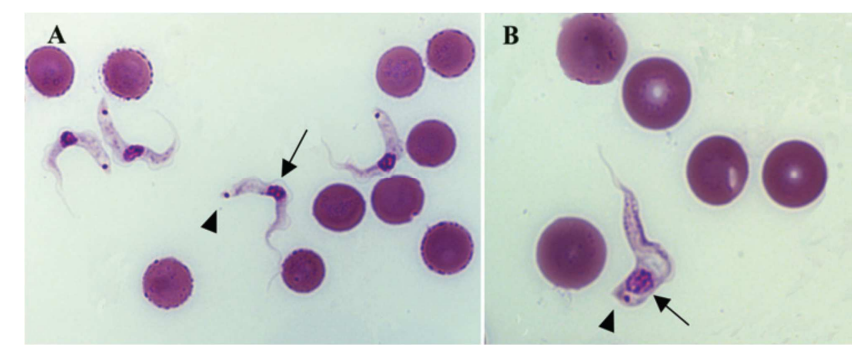

Figure 1. Giemsa staining of T. evansi bloodstream stages of long slender form (a), and akinetoplastic strain(B), indicating the nucleus (arrow) and kinetoplast (arrow head). 

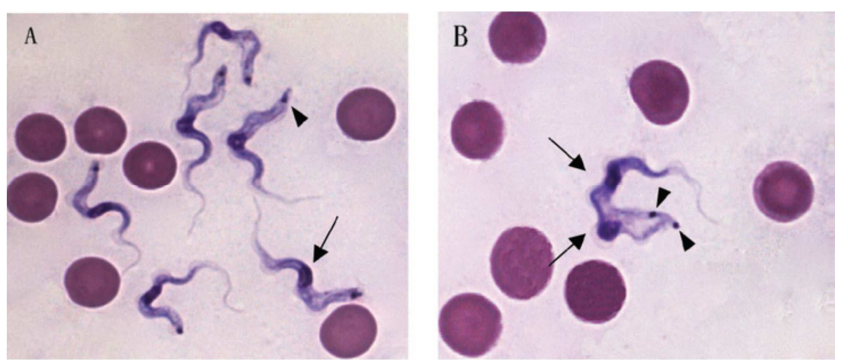

Source: (Hagose 2008).

Figure 2. Giemsa staining of T. equiperdum blood stream stage of slender form (a), and division of slender form (B), indicating the nucleus (arrow) and kinetoplast (arrow head).

\subsection{Epidemiology}

\subsubsection{Host Range}

Dourine mainly affects horses, donkeys and mules. Positive CF tests have been obtained from zebras, although it has not been shown that zebras can be infected with $T$. equiperdum or transmit the disease. The organism has been adapted to a variety of laboratory animals [13]. Improved breeds of horses seem to be more susceptible to the disease and milder in native ponies, donkeys and mules [14].

Horses are very susceptible to T. equiperdum than other equines. The disease in these animals often progresses rapidly and involves the nervous system. They usually die at the end of the chronic disease that may last for $1-2$ years. Donkeys and mules, despite being susceptible to infections, develop a mild syndrome or remain a symptomatic. Infected male donkeys, which may be asymptomatic, are particularly dangerous in the epidemiology of the disease, for they may escape detection as carriers. Horse and donkey appear to be the only natural reservoir of T. equiperdum [14].

Various laboratory animals including rabbits, rats and mice can be infected experimentally. Dourine signs have been reported in sheep and goats that were inoculated with a murine-adapted strain, but ruminants do not seem to be susceptible to the isolates from squids [15].

\subsubsection{Geographic Distribution}

Dourine is a veneraly transmitted disease caused by $T$. equiperdum that exclusively affects equines and has a wide geographic distribution. The disease is endemic in North and South Africa, the Middle east, Eastern Europe, South America and Indonesia [15].

The latest official reports (i.e. CFT positive cases) dourine were in China, Kazakhstan, Pakistan, land Brazil, Italy, Germany, Ethiopia, Botswana, Namibia and South Africa.

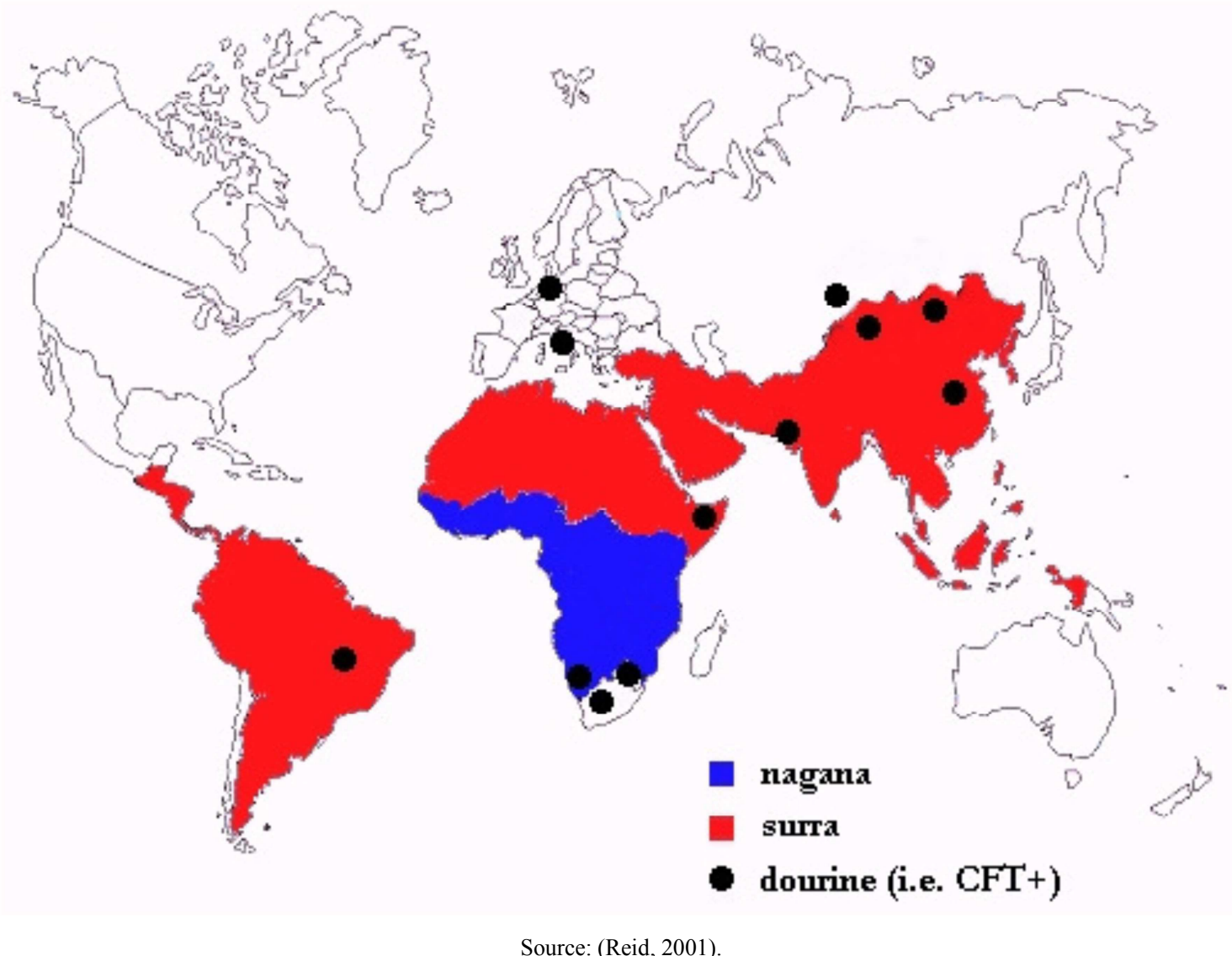

Figure 3. World Distribution of Dourine.

In Ethiopia the problem of dourine has been recognized by local farmers for many years but the first official report of the disease was made in 1980 when the Arsi Rural Development Unit requested the tsetse and trypanosomosis survey and 
control department to investigate a persistent disease problem in horses in the administrative regions of Arsi and Bale. Since then, some reports were made on the occurrence of dourine in these zones. This study focused on a thorough determination of the prevalence, extent and distribution of dourine in horses of the Arsi-Bale highlands [1].

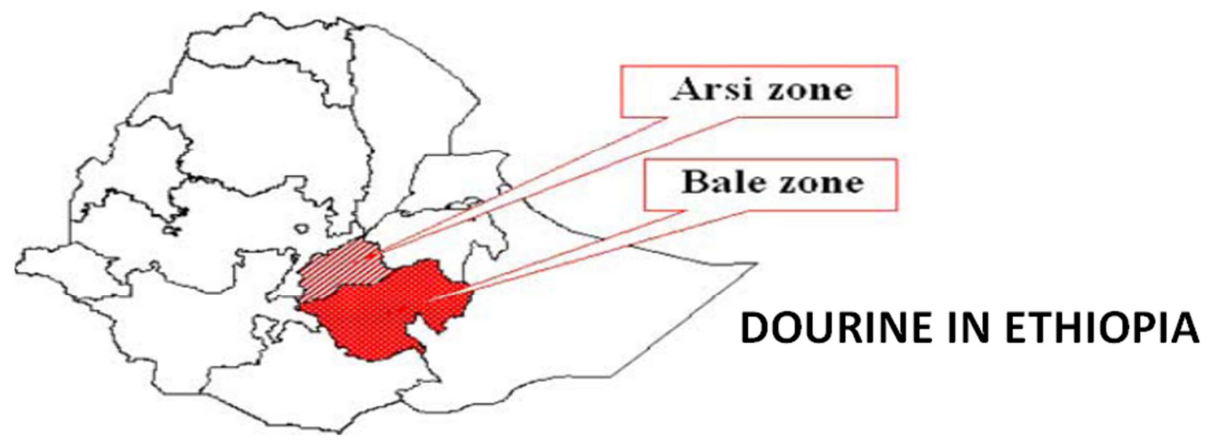

Source: (Hagos, 2005).

Figure 4. Distribution of Dourine in Ethiopia.

According to the ARDU report, the disease was widely spread in Ethaya, Sagure, Bekoji and Koffle districts of Arsi-Bale highlands as the experiment conducted is confirmed by D/r Hagos as shown in the following table.

Table.2. Seroprevalence of dourine in horses of the Arsi-Bale highlandsBased on CATT, LATEX and ELISA test.

\begin{tabular}{|c|c|c|c|c|}
\hline \multirow{2}{*}{ Study districts } & \multirow{2}{*}{ Sample size } & \multicolumn{3}{|c|}{ Sero-prevalence } \\
\hline & & CATT & LASEX & ELISA \\
\hline Arsi-Robe & 128 & $20(15.63 \%)$ & $17(13.28 \%)$ & $13(10.16 \%)$ \\
\hline Assassa & 130 & $36(27.69 \%)$ & $34(26.15 \%)$ & $26(20 \%)$ \\
\hline Koffle & 128 & $49(38.28 \%)$ & $46(35.94 \%)$ & $36(28.13 \%)$ \\
\hline Dodola & 91 & $30(32.97 \%)$ & $26(28.57 \%)$ & $22(24.18 \%)$ \\
\hline Goba & 86 & $27(31.39 \%)$ & $18(20.93 \%)$ & $14(16.28 \%)$ \\
\hline Overallprevalence & 646 & $184(28.35 \%)$ & $161(24.81 \%)$ & $125(19.26 \%)$ \\
\hline
\end{tabular}

Source: (Hagos, 2005).

In those areas, the disease is known commonly as "Lappessa" or "Duda Kuta" which means emaciation or backbone breaker in the local language, respectively. Because of the unrestricted movement of animals throughout the country for trade and transport purpose, lack of adequate facilities for diagnosis and control of the disease in relation to breeding, there have been reports indicating the presence of T. equiperdum causing dourine in the other part of Ethiopia based on clinical signs [16], serological and molecular tests $[1,4,8]$, especially in places where there are equine populations as indicated in the table below.

Table 3. Result of dourine investigation on the basis of serological and questionnaire survey in horse from different selected representative part of Ethiopia.

\begin{tabular}{|c|c|c|c|c|}
\hline \multirow{2}{*}{ Regiog } & \multirow{2}{*}{ Zone/district } & \multicolumn{3}{|c|}{ Number of seropositive animals } \\
\hline & & CATT & LATEX & ELISA \\
\hline Amhara & Awi/Enjibara & 1 & 2 & 2 \\
\hline Amhara & South Wollo/Kutaber & 4 & 3 & 3 \\
\hline Oromia & Gurji/uraga & 18 & 12 & 14 \\
\hline Oromia & Jima/dedo & 4 & 2 & 2 \\
\hline Oromia & Ease Shoa/shashemene & 6 & 5 & 1 \\
\hline Somalia & Shinella/Shinella & 3 & 2 & 0 \\
\hline SNNPRS & Sidamo/Arbegona & 9 & 9 & 1 \\
\hline
\end{tabular}

Source: (Hagos, 2005).

\subsection{Transmission}

Unlike other trypanosomal infections, dourine is transmitted from equine to equine almost exclusively during coitus. Transmission from stallions to mares is more common, but mares can also transmit the disease to stallions. $T$. equiperdum can be found in the vaginal secretions of infected mares and the seminal fluid, mucous exudate of the penis, and sheath of stallions [17]. The organism may pass through intact mucous membranes to infect the new host. Infected 
animals do not necessarily transmit the infection with every sexual encounter. As the disease progresses, trypanosomes periodically disappear from the urethra or vagina; during these periods, the animals are non-infective. However, trypanosomes which are present in the seminal fluid and mucous membrane of the genitalia of the infected donor animals are transfer to the recipient during sexual inter course. Non infective periods may last for weeks or months and are more likely to occur in the later stages of the disease. Thus, transmission is most likely early in the disease process [17].

It is possible for mares to become infected and pregnant after mating with an infected stallion. Foals born to infected mares may be infected. It is unclear if this occurs in utero or during birth. Because trypanosomes may occur in the milk of infected mares, these foals may be infected per os during birth or by ingestion of infected milk. Foals infected in this way may transmit the disease when mature and develop a lifelong positive $\mathrm{CF}$ titer. This method of disease transmission is rare. However, some foals may acquire passive immunity from colostrum of infected mares without becoming actively infected; in such foals, the CF titer declines, and the animal becomes seronegative by 4 to 7 months of age. Although the possibility of noncoital transmission remains uncertain, it is supported by sporadic infections in sexually immature equids [7]. Trypanosomes are rarely observed in the blood stream of the host because they are normally localized in the capillary of the mucous membrane of the urogenital tract. However, a few trypanosomes occasionally appear in the peripheral blood of animal with chronic infection. This could provide the opportunity for blood sucking insect to mechanically transmit the parasite rarely [17].

Human carelessness may also be responsible for conveying the infection by using contaminated utensils and unsterilized instrument for artificial insemination. Animal other than equidae cane be infected experimentally [9].

\subsection{Morbidity and Mortality}

Although the course of the disease may be long, it is usually fatal. Uncomplicated dourine does not appear to be fatal unless the nervous system is involved. The progressive debilitation associated with the neurological manifestation of the disease predisposes infected animals to a variety of other conditions. Because of the long survival time in some experimental cases, reports of recovery from dourine should be regarded with skepticism. More severe disease is usually seen in improved breeds of horses, while donkeys, mules and native ponies tend to be more resistant [15].

The mortality rate in untreated cases is estimated to be 50$70 \%$. However, apparent recoveries have been questioned by some, in view of the long course of the disease and the waxing and waning symptoms. Some authors feel that nearly all cases are eventually fatal. In endemic areas, drug treatment may be possible; however, treatment may result in inapparent disease carriers [17].

\subsection{Pathogenesis}

T. equperdum is a specific and unique trypanosome of equine in which it causes a serious disease that the organism shows a remarkable tropism for the mucosa of the genital organs, the subcutaneous tissue and the central nerves system. It is thought that when the parasite invades the tissue they cause vasomotor disturbance with the exudation of the plasma and an inflammatory reaction at the site of irritations, giving rise to the edematous swellings and plaques. The toxin elaborated in this lesion is carried away through the blood stream, causing inflammation and degeneration of the peripheral nerves. Invasion of the nervous system leads to incoordination and paralysis [19].

The trypanosomes, which are present in the seminal fluid and mucous membranes of the genitalia of the infected donor animal, are transferred to the recipient during sexual intercourse. Parasites then may pass into the blood, where they are carried to other parts of the body. In typical cases, this metastatic invasion gives rise to characteristic cutaneous plaques. Dourine is often fatal, but spontaneous recoveries can occur [10]. The incubation period, severity, and duration of the disease vary considerably depending on the strain of the trypanosome or the general health of the horse; it is often fatal, however it was claimed that spontaneous recoveries do occur and latent carriers do exist as well [19].

\subsection{Clinical Sign}

Clinical signs vary considerably, depending on the virulence of the infecting strain, the nutritional status of the infected animal, and the presence of other stress factors. In some animals, clinical signs may not be apparent for up to several years (so-called latent infection) [19]. The incubation period is highly variable mostly from one week to six month. Clinical signs usually appear within a few weeks of infection but may not be evident until after several years. There are typically three clinical phases/stages [15]. All this signs are commonly seen in Ethiopian equines infected by dourine especially those in Arsi-Bale area [1].

\subsubsection{Primary Stage (Genital Edema)}

In stallions, the first symptoms are oedema of the prepuce and glans penis. The swelling may spread to the scrotum, perineum, ventral abdomen and thorax. Vesicles or ulcers may be seen on the genitalia, when they heal, these ulcers can leave permanent scars. Orchitis may occur and cause irritation, where the stallion constantly draws and retards the penis. Paraphimosis may also occur. Early symptoms in mares consist of vaginitis, with mucopurulent discharges. The vulva becomes oedematous; this swelling may extend along the perineum to the ventral abdomen and mammary gland. Vulvitis, vaginitis with polyuria and signs of discomfort may be seen. The genital region, perineum, and udder may become depigmented. Abortion can occur with more virulent strains [9]. 

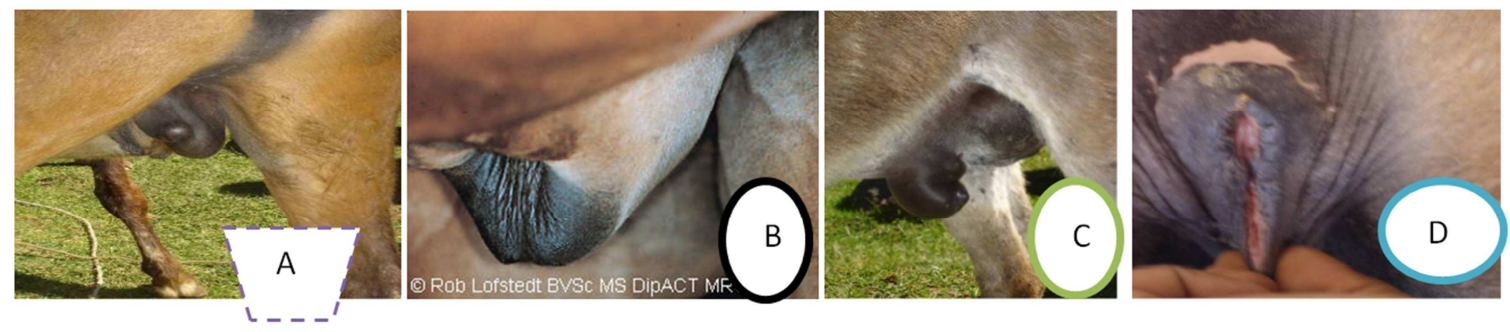

Source: (Hagos 2005).

Figure 5. A, B and C. Photos showing edematous swelling of stallion genitalia $C$, depigmentation of mares genitalia.

\subsubsection{Secondary Stage (Plaques and Skin Eruptions)}

This stage, known as stage of Urticaria, is marked by distinct, raised round or oval shaped patchy eruptions called "plaques", that appear on the skin in both sexes. Oedematous patches, also called "silver dollar plaques", up to $5-8 \mathrm{~cm}$ diameter and $1 \mathrm{~cm}$ thick may appear on the skin, particularly over the neck, shoulders, ribs and thighs, and usually last for 3-7 days, and is considered to be pathognomonic for dourine [9].

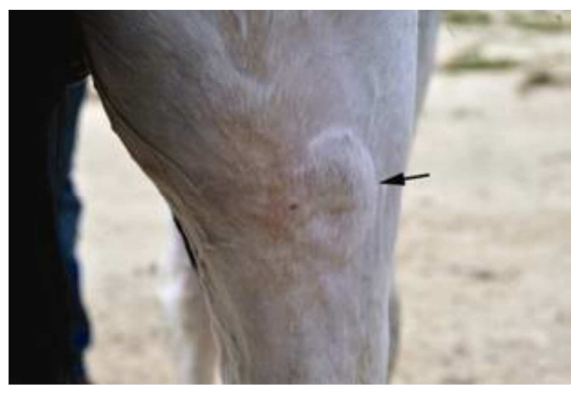

Source: (Hagos 2005).

Figure 6. Skin plaque.

\subsubsection{Tertiary Stage (Neurologic Sign)}

The final phase known as stage of paralysis is characterized by disorders of the nervous system. Initially these signs consist of restlessness and the tendency to shift weight from one leg to another followed by progressive weakness and incoordination, and ultimately, paralysis (mainly of the hind legs) and death occur. Other clinical signs include progressive anaemia seen by increasing pallor of the mucous membranes of the eyes and mouth, conjunctivitis, keratitis, intermittent fever and emaciation $[10,11]$

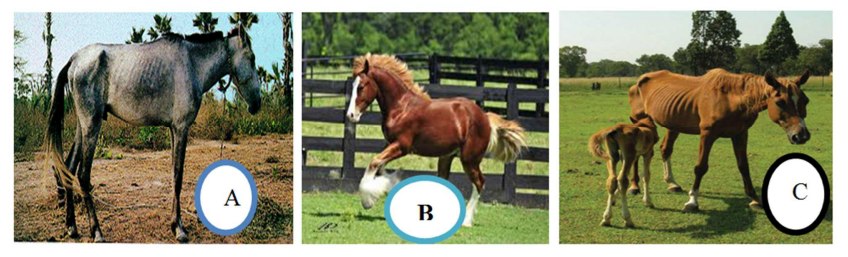

Source: (Stephen, 1996).

Figure 7. A and B, photos showing poor body condition C, photos showing leg paralysis.

\subsection{Immune Mechanism}

Infection with $T$. equiperdum may run a symptomless course in native free-range horses and horses of different breeds, as well as individual animals vary in their susceptibility to the disease [9]. Horses are very susceptible to T. equiperdum and usually die at the end of a chronic disease that may last for $1-2$ years. Donkeys and mules, despite being susceptible to infections, develop a mild syndrome or remains asymptomatic [15]. Infected animals produced antibodies to successive antigenic variants. It was also demonstrated that considerable degree of immunity conferred in utero during the last 3 month of gestation, protected a donkey foal from infection with T. equiperdum at 8 age [18].

\subsection{Pathology}

Anaemia, cachexia and genital oedema are often seen at post-mortem. The oedema, which may be indurated, can extend to the ventral abdomen. Gelatinous exudates can often be seen under the skin. In stallions, the scrotum, sheath, and testicular tunica may be thickened and infiltrated. In mares, a gelatinous infiltrate may thicken the vulva, vaginal mucosa, uterus, bladder, and mammary gland. The lymph nodes, particularly in the abdominal cavity are hypertrophied, softened and in some cases, haemorrhagic [9].

The perineural connective tissue can be infiltrated with edematous fluid, and the spinal cord may be surrounded by a serious infiltrate. A soft, pulpy or discolored spinal cord may be noted, particularly in the lumbar or sacral regions [20].

\subsection{Diagnosis}

The tentative diagnosis of diseases dourine caused by $T$. equperdum depends on the observation of clinical signs and symptoms. However, as the clinical signs are not consistent and vary with species of the host, developmental stages of the parasites as well as course of the disease, diagnosis requires confirmation by parasitological, serological and molecular techniques [21].

\subsubsection{Symptomatology}

The symptoms of edematous or dollar spots, eruption of cutaneous plaque and locomotors disturbance of hind limb are sufficiently diagnostic. The clinical disease is typical in endemic areas to allow diagnosis [22].

\subsubsection{Parasitological Diagnosis}

A definitive diagnosis of equine trypanosomosis depends on the recognition of the clinical signs followed by detection 
of trypanosomes from the blood of infected animals which is the only gold standard diagnostic test available. A number of parasitological techniques are available for the diagnosis of equine trypanosomosis [20].

Wet and thick blood films

In this test 5 to $10 \mu \mathrm{l}$ of blood is placed on a slide and examined microscopically at x400 magnification under a coverslip. Trypanosomes are observed moving between the erythrocytes in infected animals. It has very low sensitivity, with a detection limit as high as 10,000 trypanosomes $/ \mathrm{ml}$, but it is still in use because of its low cost and simplicity. Giemsa or Field's-stained thin blood films have a similarly low sensitivity. Examination of $20 \mu \mathrm{l}$ of stained blood in a thick blood film slightly improves sensitivity, with a detection threshold of around 5,000 trypanosomes $/ \mathrm{ml}$ of blood. It is time consuming (10-20 minutes per slide) and requires expertise to recognize the parasite [23].

\subsubsection{Micro Hematocrit Centrifugation Technique (mHCT)}

Micro Hematocrit Centrifugation Technique (mHCT) is a blood concentration technique (also called the capillary tube centrifugation technique or the Woo test) which was developed 40 years ago and is still the most frequently applied concentration technique with better sensitivity than direct microscopic examination. In this test, capillary tubes containing anticoagulant are filled three-quarters full with blood. The dry end is sealed with plasticine. By high-speed blood centrifugation in a hematocrit centrifuge for 6 to 8 minutes, trypanosomes are concentrated between the red blood cells and the plasma, together with the white blood cells. The capillary tubes mounted in a special viewing holder, can be directly examined at low magnification (x10 or $\mathrm{x} 40)$ for motile parasites. The estimated detection threshold of $\mathrm{mHCT}$ is 500 trypanosomes $/ \mathrm{ml}$ of blood sample [24].

\subsubsection{Mini Anion-Exchange Centrifugation Technique (mAECT)}

The mini Anion-Exchange Centrifugation Technique (mAECT) consists of separating the trypanosomes, which are less negatively charged than blood cellular components from venous blood via anion-exchange chromatography and finally concentrating them at the bottom of a plastic collector tube by low speed centrifugation. The tip of the glass tube is then examined in a special holder under the microscope for the presence of trypanosomes. The large blood volume up to $300 \mu$ enables the detection of less than 100 trypanosomes/ml, resulting in high sensitivity. However, the manipulations are quite tedious and time consuming [24].

\subsubsection{Animal Inoculation}

Inoculation of laboratory animals with blood and genital washes from antigenaemic horses was used as a supporting method of diagnosis. However, in a recent report, blood and genital washes form antigenaemic horses did not lead to infections when inoculated in to mice [4].

T. equiperdum is an extremely difficult organism to establish in laboratory animals such as mice and rats. The most successful method developed to date is the inoculation of rabbit testes with contaminated material from horse. The parasite can be maintained by serial intra testicular passage. The infection can also be transmitted to ruminants, which display mild classical manifestations. Those laboratory strains, which have been established in rodents, display antigenic variation similar to other trypanosomes stocks [5].

\subsubsection{Serological Techniques}

Diagnosis of $T$. equiperdum by standard parasitological techniques is difficult, owing to the low numbers of parasites in the blood or tissue fluids. Consequently, the demonstration of trypanosomal antibodies in the serum has become the most important parameter determining the disease status of individual animals [25]. Enzyme linked immunosorbent assay is a very sensitive technique. The use of ELISA for routine diagnosis of dourine would provide a significant advantage over current serological tests if a defined antigen were used, since it would permit test standardization and more readily allow comparison of tests among laboratories. It additionally, lends itself to a considerable degree of automation, which makes it suitable for large number of samples [26]. Different workers have stated that the ELISA has a satisfactory concordance ratio with CFT and can be used to supplement CFT [4, 27].

The complement fixation test is the most commonly used OIE prescribed serodiagnostic test developed for $T$. equiperdum 80 years ago and successfully used as part of a program to eliminate T. equiperdum from North America. It is still used for international trade in monitoring horses to export / import. Despite the usefulness and universal acceptance of the CFT for diagnosing dourine, some discrepancies have been recorded. The disadvantages of the CFT are that it requires careful continuous titration of numerous labile agents and that it does not function with sera having anti-complementary activity. CFT is not species specific, but only specific for the subgenus Trypanozoon. The drawback of the test is lower specificity where it cannot differentiate T. equiperdum from other similar trypanosomes. Hence, the diagnostic significance of CFT is therefore doubtful in countries where both T. equiperdum and T. evansi infection occur in equines [14]. Although the CFT has been in use for many years for diagnosis of dourine, it is considered to be less sensitive than ELISA and IFAT for the detection of the serum antibodies against T. equiperdum [25, 26].

Indirect fluorescent antibody test is frequently used for the diagnosis of dourine, as a confirmatory test for CFT results, since immunofluorescence provides a reliable and sensitive technique. But its interpretation is both subjective and labour intensive and it is therefore more suited to the testing of small numbers of sera [27].

The differential diagnosis includes coital exanthema, surra, anthrax, equine infectious anemia, equine viral arteritis and causes of purulent endometritis such as contagious equine metritis [12]. 


\subsection{Treatment and Control Aspect of Dourine}

The different distributions of the trypanosomes in the body of the host result in varying susceptibilities to trypanocides depending on their pharmacodynamics (mechanisms of drug action) and pharmacokinetics (disposition and fate of drugs in the body). Relapse of infection, i.e., return of patent parasitemia after apparent cure where parasites disappear from the circulation but, after a period of weeks they reestablish themselves in the circulation. The relapse might be due to the appearance of trypanosome populations from privileged sites, such as the cerebrospinal fluid and/or intercellular tissue spaces. There are no officially approved drugs to treat horses suffering from dourine. But recommended treatments are Berenil (Diminazene aceturate) at a dose of $7 \mathrm{mg} / \mathrm{kg}$ body weight as a $5 \%$ solotion injected intramuscularly with a second injection of half the dose 24 hours later, melarsomine, isometamidium chloride, suramin sodium $(10 \mathrm{mg} / \mathrm{kg}$ intravenously for two to three treatment at a weekly intervals) and neoarsphenamine [23, 28, 29].

The antitrypanosomal drug melarsamine hydrochloride (Cymelarsan, Merial) is made by conjugation of one equivalent of melarsen oxide and two equivalents of cysteamine. This water-soluble trivalent arsenical agent, Cymelarsan, has been shown to be very effective against $T$. $b$. brucei, T. evansi in camels, buffalo, goats, and pigs and against $T$. equiperdumin vitro and in vivo [28, 30, and 31]. On the basis of those experiments this compound has been licensed for use against trypanosomosis in animals. Cymelarsan is rapidly absorbed following intramuscular injection and is cleared from the circulation within 6 hours. It is also said to cross blood brain barrier (BBB) which may be effective in preventing relapses often seen after treatment with other trypanocides [32].

Table 4. Trypanocidal drugs available for the treatment of trypanosomosis in domesticanimals.

\begin{tabular}{lll}
\hline Drug & Dose & Mechanism of action \\
\hline Ethidium (Boots) & $1 \mathrm{mg} / \mathrm{kg}(\mathrm{IM})$ & Interferes with nucleic acid synthesis by intercalative DNA binding \\
Berenil (Intervet) Diminasan & $3.5-7 \mathrm{mg} / \mathrm{kg}(\mathrm{SC}$ or IM) & Intercalate Kinetoplast DNA (interferes with nucleicacid synthesis) \\
Samorin (Merial) Trypamidium (Merial) & $0.25 \mathrm{to} 1 \mathrm{mg} / \mathrm{kg}($ Deep IM) & Cleavage of kDNA topoisomerase complexes \\
Naganol (production banned) & $10 \mathrm{mg} / \mathrm{kg}(\mathrm{IV})$ & Interfere with energy metabolism of the parasites \\
Trypacide(Imperial Chemical Pharmaceutical Ltd.) & $5 \mathrm{mg} / \mathrm{kg}(\mathrm{SC})$ & Interfere with energy metabolism of the parasites \\
Cymelarsan (Merial) & $0.25 \mathrm{mg} / \mathrm{kg}$ (Deep IM) & Interfere with energy generation of the parasites \\
\hline
\end{tabular}

Source: (FAO, 1998; Uilenberg, 1998).

The only effective control of dourine remains strict control of breeding and good hygiene are essential during assisted mating because infection may be transmitted through contaminated fomites. Serological testing ensures that infected animals for breeding and legislation requiring testing of horse for export prevents the movement of infected animals [3]. In dourine free countries, an embargo should be placed on the importation of horses from countries where the disease is enzootic. In enzootic areas, the disease can be eradicated on an area or herd bases by the application of the CFT along with strict control of breeding and movement of horses [19].

Generally the most successful prevention and eradication programs depend upon: serologic identification of chronic carrier animals by the CFT and slaughter or castration of positive stallions to prevent further transmission of the disease, reeding and movement of horses should be strictly controlled, all equids in an area where dourine is found should be quarantined and breeding should be stopped for 1 to 2 months while testing continues. It is customary to treat all mares with Berenil at the time of mating. In-contact animals are considered free after three consecutive negative complement fixation tests at monthly intervals. Good Stable hygiene is always recommended. Any horse testing positive will be required to remain under official breeding and movement restrictions indefinitely. Any venereal contacts with confirmed infected horses must be isolated and will be blood tested to determine the presence of parasite Infected animals should be humanely destroyed or castrated to prevent further transmission of the disease. There are no vaccines available

\section{Conclusion and Recommendations}

In African countries mainly in Ethiopia where the disease is endemic and of significance importance given the indispensable role played by equines, the disease deserves more attention. The great economic significance of equine can only be exploited if dourine, which has been found to be a potential threat to the life and productivity of the equine population, is well conducted.

Diagnosis of T.equiperdum, the causative agent of dourine in horse by standard parasitological techniques is difficult owing to the low number of parasite present in blood or tissue fluids. Consequently, the demonstration of trypanosomal antibodies in the serum has become the most important parameter in determining the disease status of individual animals.

Dourine which his to be default known as a disease occurring only in the Arsi-Bale high land is getting spreading and becoming a potential threat to equines in the geographically adjacent areas through trade, unrestricted movement and breeding of equine (horse) outside of endemic foci areas of the country.

Based on the above remarks, the following recommendations are for warded: A special training for the horse's stockholders should be given about the importance of the dourine, awareness establishment in strict control of breeding and movement of dourine cases horses, conduct most sensitive serological and molecular technique for diagnosis, develop compulsory slaughter of infected stallions 
and mares as a national policy and needs further more research on specific control and treatment of dourine.

\section{References}

[1] Hagos A. (2005): Serological and parasitological survey of dourine (Trypanosoma equiperdum) in selected sites of Ethiopia. MSc Thesis, Addis Ababa University, Faculty of Veterinary Medicine, Debre Zeit, Ethiopia. Pp: 1-270.

[2] Food and Agriculture Organization (FAO) of the United Nations., (1998): Drug management and parasite resistance in bovine trypanosomosis in Africa. ISBN, Pp 92-97.

[3] Zablotskij, V., Georgiu, C., De Waal, T., Clausen, P., Claes, F., Touratier, L., (2003): The current challenges of dourine: difficulties in differentiating Trypanosoma equiperdum within the subgenus Trypanozoon. Rev. Sci. Tech. Off. Int. Epizoot. 22: $1087-1096$

[4] Alemu T, Luckins A, Philips L, Reid S \& Holmes P. (1997): The use of ELISA to investigate theprevalence of Trypanosomes equiperdumin Ethiopian horses. Vet. Parasitol., 71: 239-250.

[5] Molyneux, D. and Ashiford, R., (1993): The biology of trypanosome and Leshimania, parasites of man and domestic animal. Taylor and Francis Ltd. UK, Pp. 158-160.

[6] Hagos, A., Yilkal, A., Esayass, T., Fikru, R., Feseha, G/ab., Goddeeris, B., Claes, F, (2009): Parasitological and serological survey on surra in camels in dry and wet areas of Bale Zone, Oromia Region, Ethiopia. Revue Med. Vet. 160: 569-573.

[7] Kahn CM, Line S, editors (2006): The Merck veterinary manual, Whitehouse Station, NJ: Merck.

[8] Clausen P., Gebreselassie G., Abditcho S., Mehlitz D. \& Staak C. (1999): Detection of trypanosoma DNA in serologically positive but aparasitaemic horses suspected of dourine in Ethiopia. Tokai. J. exp. clin. Med., 23, 303-308.

[9] OIE, (2008): Manual of Standards for Diagnostic Tests and Vaccines for Terrestrial Animals. $6^{\text {th }}$ edition, OIE Publications, Paris.

[10] Stephen, L., (1996): Trypanosomiasis: a veterinary perspective, Pregamon Press, Oxferd, UK. Pp. 216-224.

[11] Hoare C, (1992): The Trypanosomes of Mammals. Blackwell Scientific Publications Oxford.

[12] Vanhamme L, paturiauxHanocq F, poelvoorde pet al., (2003): apolipoprotein L-is the trypanosome lytic factor of human serum. 422: 83-87.

[13] Claes F., Ilgekbayeva G., Verloo D., Saidouldin T., Geerts S., Buscher P. \& Goddeeris B. (2005): Comparison of serological tests for equine trypanosomosis in naturally infected horses from Kazakhstan. Vet. Parasitol., 131,. 221-225.

[14] Luckins, A. G., (1994): Equine Trypanosomosis. Eq. Vet. Educ. 6: 259-262.

[15] Maudlin, I., Dieter, M., Susan, C., (2004). The relationship between rickettsia-like-organisms and trypanosome infections in natural populations of tsetse in Liberia. Tropical medicine and parasitology: official organ of Deutsche Tropenmedizinische Gesellschaft and of Deutsche
Gesellschaft für Technische Zusammenarbeit (GTZ) 41(3): 265-7 October 1990 with 34 Reads

[16] Zeleke D., Ketema S. \& Abdul S., (1980): An investigation of dourine in Arsi Administrative Region.

[17] Brun r, Hecker H, Lun Zr., (1998): T. evansi and $T$. equiperdum: distribution, biology, treatment and phylogenetic relationship (a review). Vet parasitol, Pp. 95-107.

[18] OIE (2001): Dourine. Part2. In:Manual of standards for diagnostic test and vaccines, $4^{\text {th }}$ editon, OIE publication, Paris, Pp. 128-5

[19] Radostits, O., Blood, D., Gay, C., (1994): Veterinary Medicine; A text book of the disease of cattle, sheep, pigs, goat and horses. $8^{\text {th }}$ ed., Baillere Tindall, UK, Pp. 1220-1225.

[20] Joshi pp, Shegokar Vr, powar rM et al, (2005): Human trypanosomiasis caused by trypanosomaevansi in india: the first case report. Am J Trop Med Hyg; 73:491-495.

[21] Gibson, W., ( 2007): Resolution of the species problem in African trypanosomes. J. Int. Parasitol. 37: 829-838.

[22] Kaufmann, J. (1996): Parasitic infections of domestic animals, a diadnistic mauwal. Klwauser Vetlage, Berlin. Pp. 233-234.

[23] Lun Zr, Brun r, Gibson W.(1992): Kinetoplast dNaand molecular karyotypes of trypanosoma evansi and trypanosoma equiperdum from china. Mol Biochem parasitol, 50: 189-196.

[24] Reid Sa, (2002): trypanosoma evansi control and containment in australasia. trends parasitol, Pp. 219-224.

[25] Bishop, P., Rae, P., Philips, L., Boid, R., Luckins, A., (1995): Trypanosoma equiperdum: Detection of Trypanosomal antibodies and antigen by enzyme- linked immunosorbent assay. J. Br. Vet., 151: 715-720.

[26] Wassal, D., Gregory, R., Phipps, L., (1991): Comparative evaluation of enzyme- linked immunosorbent assay (ELISA) for the serodiagnosis of dourine. Vet. Parasitol, 39: 233-239.

[27] Williamson, C., Stoltz, W., Mattheus, A., Schiele, G., (1988): An investigation into alternative methods for the serodiagnosis of dourine. Onderstepoort J. Vet. Res., 55:117-119.

[28] Zhang, Z., Giroud, C., Baltz, T., (1992): In vivo and in vitro sensitivity of $T$. evansi and T. equiperdum to Diminazene, Suramine, Melcy, Quinapyramine and Isometamidium. Acta Trop. 50: 101-110.

[29] Kaminsky, R., Schmid, C., Lun, Z. R., (1997): Susceptibility of dyskinetoplastic $T$. evansi and $T$. equiperdum strains to Isometamidium Chloride. Parasitol. Res. 83: 816-818.

[30] Zweygarth, E., Kaminsky, R., (1990). Evaluation of an arsenical compound (MelCy, Cymelarsan) against susceptible and drug-resistant Trypanosoma brucei brucei and $T$ b. evansi. Trop Med Parasitol, 41: 208-212.

[31] Payne, R., Sukanto, I., Partoutomo, S., Jones, T., Luckins, A., Boid, R., (1994): Efficacy of Cymelarsan ${ }^{(B)}$ in Friesian Holstein calves infected with Trypanosoma evansi. Trop. Anim. Hlth. Prod. 26: 219-226.

[32] Raynaud, J., Sones, K., Friedheim, E., (1989): A review of Cymelarsan - a new treatment proposed for animal trypanosomiasis due to $T$. evansi and other trypanosomes of the T. brucei group. ISCTRC, $20^{\text {th }}$ Meeting, Mombasa, Kenya. 